\title{
Maturidade sexual fisiológica em Goniopsis cruentata (Latreille) (Crustacea, Brachyura, Grapsidae) no Estuário do Paripe, Pernambuco, Brasil
}

\author{
Noely Fabiana Oliveira de Moura \& Petrônio Alves Coelho
}

\author{
Laboratório de Carcinologia, Departamento. de Oceanografia, Universidade Federal de Pernambuco. Avenida Arquitetura, \\ Cidade Universitária, 50790-540 Recife, Pernambuco, Brasil.
}

\begin{abstract}
Physiological sexual maturity of Goniopsis cruentata (Latreille) (Crustacea, Brachyura, Grapsidae) in the Paripe Estuary, Pernambuco State, Brazil. This study analyzed physiological sexual maturity of Goniopsis cruentata (Latreille, 1803) in the Paripe Estuary, Pernambuco State, Brazil. Crabs were sampled monthly from August 1999 to July 2000, with a specific fishing gear, with one hour effort per sampling period.After sampling, all individuals were sexed; the carapace width $(\mathrm{CW})$ was measured and organism it was dissected to observe the macroscopic gonad development. A total of 664 specimens were sampled (324 males, 228 females without eggs and 112 ovigerous) with predominance of sexually mature individuals for both sexes. The male's size (CW 50\%) was $31.64 \mathrm{~mm}$ and female's one was $28 \mathrm{~mm}$. The smallest ovigerous female found had a $30.5 \mathrm{~mm}$. For $G$. cruentata it was observed a synchronicity at the beginning of the sexual maturity for both sexes. This fact is usually observed among Brachyura, mainly the Grapsidae.
\end{abstract}

KEY WORDS. Reproduction, synchronicity sexual.

RESUMO. O presente estudo analisou a maturidade sexual fisiológica de Goniopsis cruentata no estuário do rio Paripe, Pernambuco, Brasil. Os caranguejos foram amostrados mensalmente de agosto 1999 a julho de 2000, com uma aparelho de pesca artezanal e com esforço de pesca de uma hora. Depois da amostragem, todos os indivíduos foram sexados, a largura da carapaça (LC) foi medida e o organismo dissecado para análise macroscópica das gônadas. Foram analisados um total de 664 espécimens (324 machos, 228 fêmeas sem ovos e 112 ovígeras) com a predominância de indivíduos maturos para ambos os sexos. O tamanho em que $50 \%$ dos machos apresentam-se fisiologicamente maturos na população amostrada é de $31,64 \mathrm{~mm}$ e as fêmeas com $28 \mathrm{~mm}$. A menor fêmea ovígera encontrada mediu 30,5 mm. Para G. cruentata foi observado uma sincronia no início da maturidade sexual para ambos os sexos. Este fato é usualmente encontrado entre os Brachyura, particularmente entre grapsídeos.

PALAVRAS CHAVES. Reprodução, sincronia sexual.

A determinação do tamanho em que ocorre o início da maturidade sexual dos siris e caranguejos é de extrema importância na implantação de planos de manejo em espécies com valor comercial ou alimentar (AnNala et al. 1980). De acordo com KNUCKEY (1996), várias espécies de caranguejos exploradas comercialmente tem citado em seu plano de manejo o tamanho mínimo de captura, garantindo que a população possa atingir a maturidade e possam se reproduzir pelo menos uma vez. Como exemplo pode ser observado no caranguejo Ucides cordatus (Linnaeus, 1763) no litoral nordestino, onde a captura das fêmeas é proibida, enquanto os machos podem ser extraídos comercialmente a partir de um tamanho mínimo de $45 \mathrm{~mm}$ de largura cefalotorácica (Ivo \& Gesteira 1999).

O tamanho de maturidade sexual em braquiúros tem sido determinado de várias formas, baseando-se em análises de maturidade morfológica, critérios de crescimento relativo; maturidade fisiológica, a partir de observações sobre a maturação gonadal; e maturidade funcional, em observações comportamentais da corte e cópula (HaRTNOLL 1974, SAMPEDro et al. 1997). Variações intraespecíficas no tamanho da maturidade sexual de braquiúros têm sido relatada tanto local (Díaz $\&$ Conde 1989) como geograficamente (Hines 1982), sendo associadas a fatores exógenos como a temperatura e disponibilidade de alimento (ANNALA et al. 1980, WENNER et al. 1974).

Goniopsis cruentata (Latreille, 1803) se distribui no Atlântico Ocidental, desde a Flórida (EUA) até próximo ao Estado de Santa Catarina (Brasil) (Melo 1996). É um caranguejo que habita áreas de manguezal, ocorrendo em substratos consolidados e inconsolidados do supra e mediolitoral (Coelho 1965, Coelho \& Coelho-Filho 1993, Coelho \& Ramos-Porto 1995). Trata-se de uma espécie abundante nos estuários de Pernambuco, Nordeste do Brasil, onde se destaca por seu valor econômico, constituindo importante fonte de renda para as populações ribeirinhas. O tamanho de maturidade morfológica de $G$. 
cruentata já foi relatado para a região sudeste do Brasil, por Cobo \& Fransozo (1998) e Silva \& Oshiro (2002), no litoral norte paulista e Rio de Janeiro, respectivamente. No entanto, para a região nordeste brasileiro, onde a espécie é intensamente explorada, não há registro de estudos sobre o tamanho no início da maturação sexual. Desta forma, o presente trabalho tem por objetivo determinar o tamanho no início da maturidade fisiológica do caranguejo G. cruentata, para uma população ao norte do Estado de Pernambuco, litoral nordeste do Brasil.

\section{MATERIAL E MÉTODOS}

As coletas foram realizadas mensalmente no período de agosto/1999 a julho/2000, ao longo do estuário do Rio Paripe, Itamaracá, Pernambuco, Brasil. Os animais foram coletados com o auxílio de uma vara com linha de nylon, em cuja extremidade era colocada uma isca constituída por músculos de peixe, pele da galinha ou caranguejos menores, como Aratus pisonii (H. Milne Edwards, 1837) e Uca spp. Após a coleta, os indivíduos foram acondicionados em sacos plásticos, transportados até o laboratório e mantidos sob congelamento até o momento das análises.

Os exemplares foram separados por sexo, mensurados com paquímetro de precisão de $0,001 \mathrm{~mm}$ (LC = largura da carapaça). Posteriormente, foram dissecados para análise macroscópica das gônadas quanto a sua coloração e tamanho em relação a glândula do intestino médio (hepatopâncreas) e cavidade cefalotorácica.

Foram definidos quatro estágios de maturação gonadal para as fêmeas, modificados de Choy (1988), Abelló (1989), CHU (1999) e Mantelatto \& Fransozo (1999), a saber: Imatura (IM), gônadas não diferenciadas sob estereomicroscópio, geralmente associadas a indivíduos jovens; Rudimentar (RU), ovário filamentoso, detectável sob esteriomicroscópio e com coloração esbranquiçada ou amarela clara; Em desenvolvimento (ED), ovário com cerca de 1/4 do tamanho do hepatopâncreas, apresentando superfície externa rugosa e coloração alaranjada; Desenvolvida (DE), com o ovário ocupando cerca de $1 / 2$ do volume do hepatopâncreas e tendo coloração parda, um indicativo da condição de pré-desova e Condição de Ovígera, fêmeas com presença de massa de ovos (OV). Para os exemplares machos, as gônadas foram classificadas em apenas dois estágios: Imatura (IM), gônadas não diferenciadas sob esteriomicroscópio; e Matura (MA), gônadas filamentosas e de coloração branco leitosa.

O tamanho da maturidade sexual fisiológica foi determinado após a distribuição dos indivíduos em classes de tamanho e confecção de uma curva logística demonstrando o tamanho estimado em que $50 \%$ dos indivíduos de cada sexo estão fisiologicamente maduros.

\section{RESULTADOS}

Foram examinados 664 exemplares, 324 machos (49\%), 228 fêmeas sem ovos (34\%) e 112 (17\%) ovígeras. O tamanho dos exemplares analisados variou de 19,3 a 55,2 mm para os machos, 20,2 a 51,0 mm para as fêmeas sem ovos e de 30,5 a 47,8 $\mathrm{mm}$ para as fêmeas ovígeras. Para os machos, a distribuição de frequência dos estágios gonadais evidenciou para as classes compreendidas entre 16 a $28 \mathrm{~mm} \mathrm{100 \%} \mathrm{de} \mathrm{indivíduos} \mathrm{com} \mathrm{gônadas}$ imaturas. O início do desenvolvimento das gônadas ocorreu a partir de $28 \mathrm{~mm}$ de LC e nas classes acima de $32 \mathrm{~mm}$ de LC todos os indivíduos machos foram considerados maturos (Fig. 1).

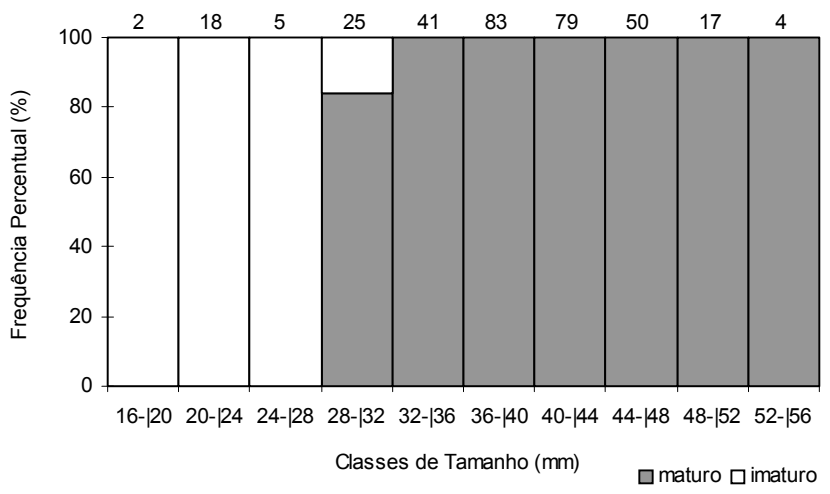

Figura 1. Histograma de distribuição de frequência dos estágios gonadais para a população de machos de G. cruentata capturados no manguezal do rio Paripe, Pernambuco, no período de agosto/1999 a julho/2000. O número acima das barras indica o número de indivíduos para cada grupo.

O ajuste da curva logística para os indivíduos machos, em classes de tamanho (4 mm) (Fig. 2) indicou 31,64 mm como o tamanho em que 50\% dos machos apresentam-se fisiologicamente maturos na população amostrada.

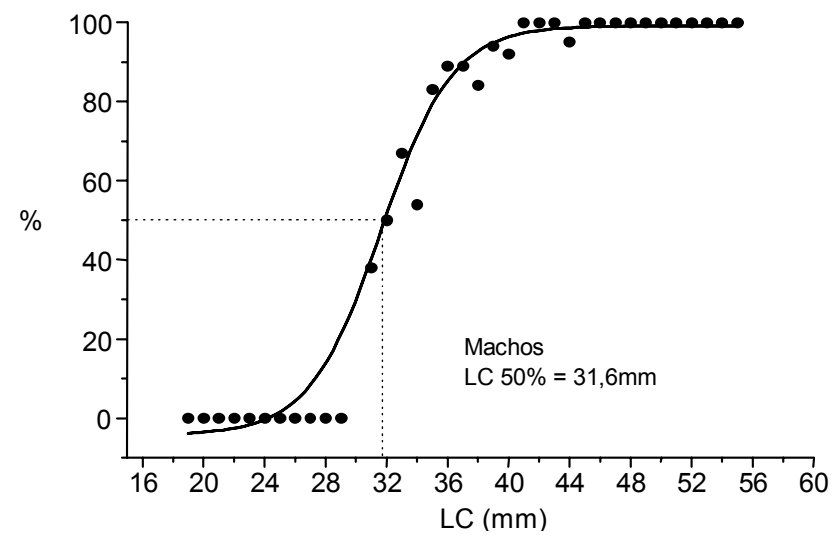

Figura 2. Porcentagem de machos com gônadas desenvolvidas de G. cruentata capturados no manguezal do rio Paripe, Pernambuco, no período de agosto/1999 a julho/2000. 
A figura 3 mostra a distribuição de frequência dos estágios gonadais das fêmeas em classes de tamanho, evidenciando de 20 a $28 \mathrm{~mm} 100 \%$ de indivíduos jovens, de 28 a $32 \mathrm{~mm}$ uma transição entre indivíduos imaturos e maturos, e a partir da classe 32 (32-| $36 \mathrm{~mm}$ ) apenas indivíduos em estágio maturo.

As fêmeas ovígeras foram registradas a partir da classe de $28 \mathrm{~mm}$, sendo que a menor mediu 30,5 mm (Fig. 3).

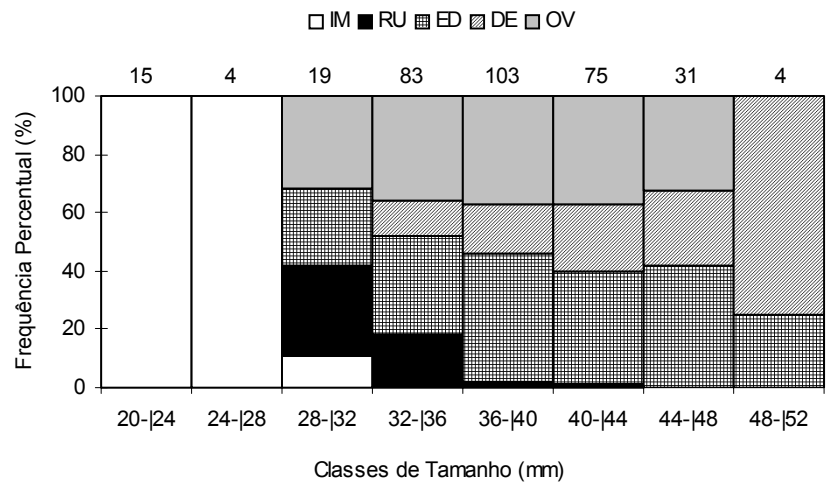

Figura 3. Histograma de distribuição de freqüência dos estágios gonadais para a população de fêmeas de $G$. cruentata capturadas no manguezal do rio Paripe, Pernambuco, no período de agosto/ 1999 a julho/2000. O número acima das barras indica o tamanho da amostra. (IM) Imatura, (RU) rudimentar, (ED) em desenvolvimento, (DE) desenvolvida, (OV) ovígera.

A partir de $28 \mathrm{~mm}$ as fêmeas iniciam a maturação gonadal, que corresponde ao tamanho estimado em que 50\% das fêmeas estão fisiologicamente maturas (Fig. 4).

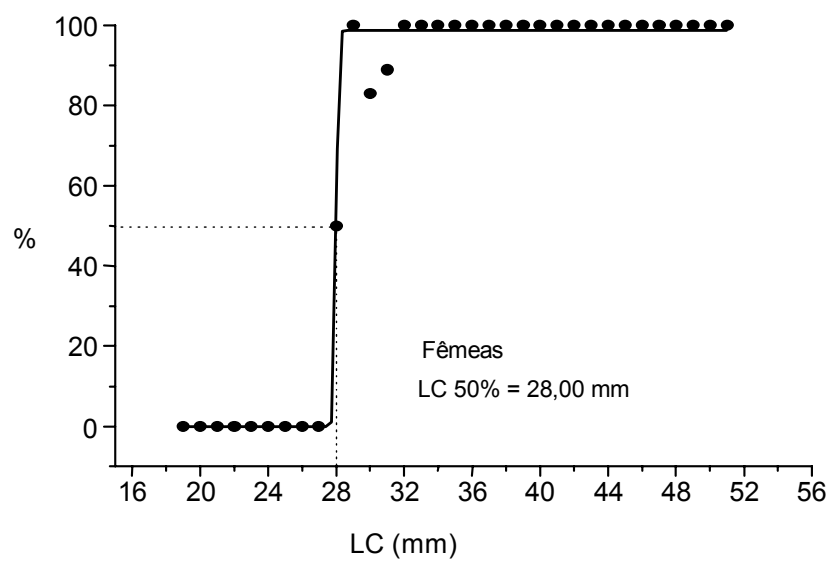

Figura 4. Porcentagem de fêmeas com gônadas desenvolvidas de G. cruentata capturadas no manguezal do rio Paripe, Pernambuco, no período de agosto/1999 a julho/2000.

\section{DISCUSSÃO}

O início da maturidade sexual representa um evento biológico importante, sendo marcado por várias transformações no animal, tanto morfológicas quanto fisiologicamente (HARTNOLL 1985). Acarreta também mudanças no papel que estes indivíduos irão desempenhar na população, influenciando novos hábitos e comportamentos. O principal evento é a transição para a fase adulta, quando passam a atuar como agentes reprodutores.

O predomínio de Goniopsis cruentata com gônadas maturas pode estar relacionado com as capturas terem acontecido sempre em dias de lua nova, pois Hagen (1970) indica que a fase lunar influencia a atividade de maturação de outros caranguejos de manguezal. Este autor observou que nas fases de lua crescente ou minguante, a maioria das espécies de Uca da Ilha de Trinidade apresentavam as gônadas imaturas ou em início de desenvolvimento, enquanto nas luas cheia e nova mais de 50\% dos espécimens apresentavam gônadas totalmente desenvolvidas.

No que se refere ao tamanho da primeira maturação, os indivíduos analisados apresentaram uma maturação tardia numa comparação com os dados obtidos por Сoвo \& Fransozo (2000) para mesma espécie, no litoral norte do Estado de São Paulo, sudeste do Brasil. Tais variações do tamanho na maturidade sexual podem ser atribuídas a diferenças latitudinais em função da atuação de fatores ambientais de distinta magnitude nas respectivas regiões, como é o caso da temperatura e sua interação com o fotoperíodo (GIESE 1959, AnNALA et al. 1980, SAMPEDro et al. 1999). Estes parâmetros exógenos podem interferir nas taxas metabólicas e no crescimento individual em populações de uma mesma espécie em regiões distintas, originando tamanhos diferenciados para o início da maturação, como observado por Hines (1989) para algumas espécies de brachyura da Costa Oeste dos Estados Unidos. Outros fatores também podem influenciar o tamanho de maturidade sexual, como a salinidade e a disponibilidade de recursos alimentares (D'INCAO et al. 1993).

Annala et al. (1980) e Armitage \& Landau (1982) mencionam que a maturidade pode ser retardada em regiões caracterizadas por baixas temperaturas ou apresentar um desenvolvimento gonadal precoce naquelas de temperaturas elevadas. Várias hipóteses podem ser levantadas sobre o retardo na maturação gonadal de G. cruentata na região de estudo, como uma diminuição na disponibilidade de alimento, estresse a condições ambientais adversas ou um favorecimento do crescimento somático. Um dos padrões relatados por WenNER et al. (1985) relaciona as espécies de crescimento rápido com uma maturidade tardia.

A sincronia sexual do tamanho no início da maturidade gonadal de G. cruentata também foi verificado por Сово \& Fransozo (1998) para a maturação morfológica da mesma espécie. Aвele et al. (1986) encontraram uma relação sincrônica de maturidade para a espécie Pachygrapsus transversus (Gibbes, 
1850), BROEKHUYSEN (1941) observou fato semelhante para maturidade fisiológica com Cyclograpsus punctatus $\mathrm{H}$. Milne Edwards, 1837. Este fato é usualmente encontrado entre os Brachyura, particularmente entre grapsídeos.

\section{AGRADECIMENTOS}

Aos amigos do Laboratório de Carcinologia do Departamento de Oceanografia da Universidade Federal de Pernambuco. Ao Conselho Nacional de Desenvolvimento Científico e Tecnológico (CNPq) e à Fundação de Amparo à Ciência e Tecnologia de Pernambuco (FACEPE). À Dra. Maria Helena Leme pela ajuda na revisão do texto.

\section{REFERÊNCIAS BIBLIOGRÁFICAS}

Abele, L.G.; P.J. Campanella \& M. Salmon. 1986. Natural history and social organization of the semiterrestrial grapsid crab Pachygrapsus transversus (Gibbes). Journal of Experimental Marine Biology and Ecology, Amsterdan, 104: 153-170.

AbelLó, P. 1989. Reproduction and moulting in Liocarcinus depurator (Linnaeus, 1758) (Brachyura, Portunidae) in the northwestern Mediterranean sea. Scientia Marina, Barcelona, 53 (1): 127-134.

Annala, J.H.; J.L. Mckoy; J.D. Booth \& R.B. Pike. 1980. Size at the onset of sexual maturity in female Jasus edwardsii (Decapoda: Palinuridae) in the New Zeland. Journal of Marine and Freshwater Research, New Zeland, 14 (13): 217-221.

Armitage, K.B. \& L.M. Landau. 1982. The effects of photoperiod and temperature on growth and reproduction of Daphnia ambigua. Comparative Biochemistry and Physiology, Oxford, 71 (A): 137-140.

Broekhuysen, G.J. 1941. The life history of Cyclograpsus punctatusi M. Edw.: breeding and growth. Transactions of the Royal Society of South Africa, Cape Town, 28: 331-336.

Сноу, S.C. 1988. Reproductive biology of Liocarcinus puber and Liocarcinus holsatus (Decapoda, Brachyura, Portunidae) from the Gower Peninsula, South Wales. Marine Ecology, Edinburgh, 9 (3): 227-241.

CHU, K.H. 1999. Morphometric analysis and reproductive biology of the crab Charybdis affinis (Decapoda, Brachyura, Portunidae) from the Zhujiang estuary, China. Crustaceana, Leiden, 72 (7): 647-658.

Сobo, V.J. \& A. Fransozo. 2000. Fecundity and reproduction period of the red red mangrove crab Goniopsis cruentata (Brachyura, Grapsidae) São Paulo State, Brazil, p. 527-533. In: J.C. von KLEIN \& F.R. Schran (Eds). The Biodiversity Crisis and Crustácea. Rotterdam, Crustacean Issues 12, 848p.

Coвo, V.J. \& A. Fransozo. 1998. Relative growth of Goniopsis cruentata (Crustacea, Brachyura, Grapsidae) on the Ubatuba region, São Paulo, Brazil. Iheringia, Série Zoologia, Porto Alegre, (84): 21-28.

Coelno, P.A. 1965. Os crustáceos decápodos de alguns manguezais pernambucanos. Trabalhos do Instituto Oceanográfico da Universidade Federal de Pernambuco, Recife, 7: 71-90.

Coelho, P.A. \& P.A. Coelho Filho. 1993. Chave para identificação dos crustáceos Decapodos Brachiuros encontrados nos biótopos de água salobra do litoral oriental do Nordeste do Brasil. Boletim Técnico-Científico do CEPENE, Tamandaré, 1 (1): 29-56.

Coelho, P.A \& M. Ramos-Porto. 1995. Distribuição ecológica dos crustáceos decápodas marinhos do nordeste do Brasil. Trabalhos Oceanográficos da Universidade Federal de Pernambuco, Recife, 23: 113-127.

D’Incao, F.; M.L. Ruffino; K.G. Da Silva; A.C. Braga \& L.H.C. Marques. 1993. Crescimento de Chasmagnathus granulata Dana, 1851, em um marisma do estuário da Lagoa dos Patos, RS (Decapoda: Grapsidae). Revista Brasileira de Biologia, Rio de Janeiro, 53 (4): 637-643.

Diaz, H. \& J.E. Conde. 1989. Population dynamics and life history of the mangrove crab Aratus pisonii (Brachyura, Grapsidae) in a marine environment. Bulletin of Marine Science, Coral Gables, 45 (1): 148-163.

GIESE, A.C. 1959. Comparative physiology: Annual reproductive cycles of marine invertebrates. Animal Review of Physiology, Stanford, 21: 547-576.

Hagen, H.O.V. 1970. Adaptations to the species intertidal level of habitat in Ocypodid crabs (Decapoda, Brachyura). Journal of Functional Biology, Germany, 2: 361-413.

HarTNOLL, R.G. 1974. Variation in growth pattern between some secondary sexual charaters in crabs (Decapoda, Brachyura). Crustaceana, Leiden, 27: 131-136.

. 1985. Growth, sexual maturity and reproductive output, p. 15-17. In: A. M. WenNER (Org.). Factors in adult growth. Rotterdam, AA. Balkema, 362p.

Hines, A.H. 1982. Allometric constraints and variables of reproductive effort in brachyuran crabs. Marine Biology, Berlin, 69: 309-320.

. 1989. Geographic variation in size at maturity in brachyuran crabs. Bulletin of Marine Science, Coral Gables, 42 (2): 356-368.

Ivo, C.T.C.\& T.C.V. GesteIra. 1999. Sinopse das observações sobre a bioecologia e pesca do caranguejo-uçá Ucides cordatus cordatus (Linnaeus, 1763) capturado em estuários de sua área de ocorrência no Brasil. Boletim Técnico-Científico do CEPENE, Tamandaré, 7 (2): 9-52.

KNUCKEY, I.A. 1996. Maturity in male mud crabs, Scylla serrata, and the use of mating scars as a functional indicator. Journal of Crustacean Biology, San Antonio, 46: 487-495.

Mantelatto, F.L.M \& A. Fransozo. 1999. Reproductive biology and moulting cycle of the crab Callinectes ornatus (Decapoda, Portunidae) from the Ubatuba region, São Paulo, Brazil. Crustaceana, Leiden, 72 (1): 63-76.

Melo, G.A.S. 1996. Manual de identificação dos Brachyura (caranguejos e siris) do litoral brasileiro. São Paulo, Plêiade, FAPESP, 604p.

Revista Brasileira de Zoologia 21 (4): 1011-1015, dezembro 2004 
Sampedro, M.P.; L. FernándeZ; J. Freire \& E. GonZÁlez-Gurriarán. 1997. Fecundity and reproductive outup of Pisidia longicornis (Decapoda, Anomura) in the Ría de Arousa (Galicia, NW Spain). Crustaceana, Leiden, 70 (1): 95-109.

Sampedro, M.P.; E. González-Gurriarán; J. Freire \& R. Muiño. 1999. Morphometry and sexual maturity in the spider crab Maja squinado (Decapoda: Majidae) in Galicia, Spain. Journal of Crustacea Biology, San Antonio, 19 (3): 578-592.

SiLVA, Z.S. \& L.M.Y. Oshiro. 2002. Crescimento em Goniopsis cruentata (Latreille, 1803) (Crustácea, Decapoda, Grapsidae) no laboratório. Revista Brasileira de Zoologia, Curitiba, 19 (3): 915-923.

Wenner, A.M.; C. Fusard \& A. Oaten. 1974. Size at onset of sexual maturity and growth rate in crustacean populations. Canadian Journal of Zoology, Ottawa, 52: 1095-1106.

Wenner, A.M.; M.P. Henry \& P.R. Siegel. 1985. Variation in size at onset of egg production, p. 149-170. In: A.M. WENNER (Ed). Factors in Adult Growth. Rotterdam, A.A. Balkema, 362p.

Recebido em 20.IV.2004; aceito em 23.XI.2004. 